\title{
Seismic data reconstruction based on Compressed Sensing
}

\author{
Xiaona Ma, Zhiyuan Li, Guanghe Liang \\ Key Laboratory of Mineral Resources \\ Institute of Geology and Geophysics, CAS \\ Beijing, China \\ maxiaona@mail.iggcas.ac.cn
}

\author{
Pei Ke \\ BGP Inc. \\ China National Petroleum Corporation \\ Zhuozhou, China \\ 602841556@qq.com
}

\begin{abstract}
In seismic exploration, seismic data is always irregular and incomplete which will influence the subsequent processing. Therefore, seismic reconstruction of missing traces is very necessary. The seismic data reconstruction method based on compressive sensing (CS) has been applied widely in recent years. Comparing to traditional seismic data gathering, CS can reduce the acquisition cost, and allows for making new seismic acquisition designs. The fast iterative threshold shrinkage algorithm (FISTA) based on Fourier transform is an effective method to construct seismic data. CS can make use of the sparse property of signal through the nonlinear to reconstruct perfectly. In this paper, we first introduce CS theory and FISTA method. Then we test the reconstruction method by numerical examples and real seismic data reconstruction. As shown in paper, the reconstruction method based on CS theory is efficient.
\end{abstract}

Keywords-seismic data reconstruction; irregular and incomplete; compressive sensing; FISTA

\section{INTRODUCTION}

In the seismic data acquisition stage, the seismic data are usually irregular and incomplete which is due to limited acquisition conditions and complex exploration, such as buildings, rivers, cliff and other obstacles located on the land. And with the restriction of exploration budget, seismic data is hardly dense; in contrast, sparse sampling is very low-priced. Irregular sampling (Fig.1 e-f) and sparse sampling (Fig.1 c-d) both influence the signal. Sparse sampling will decrease the amplitude of signal frequency spectrum, irregular sampling will lead to create alias, which would affect following data processing and interpretation. Therefore, seismic data reconstruction is important.

By now, the reconstruction methods of missing traces can be generally divided into three types: 1) Method based on math transform, such as Fourier transform and Curvelet transform; 2) Method based on convolution operator; 3) Method based on wave equation $^{[6][7-10]}$.

In recent years, David Donoho, Emmanuel Candes, Terence Tao, Romberg proposed Compressed Sensing (CS) theory ${ }^{[2][5]}$. CS indicates a sparse or compressible signal can be recovered from a small number of random linear measurements by solving a convex $l_{1}$ optimization problem, which has been used in seismic data reconstruction and survey design ${ }^{[1][4]}$.
Comparing to Compressive Sensing (CS), traditional seismic data acquisition which is based on the Nyquist sampling rate needs high requirements of gathering and storage of seismic data.

In general, seismic data are usually not sparse. Therefore, we need make use of Fourier transform to make seismic data transform from time-space domain to frequency-wavenumber domain in which the seismic data are sparse.

Moreover, fast iterative shrinkage-thresholding algorithms based on the Fourier transform is widely applied for reconstructing irregularly sampled seismic data, due to its simplicity, practicability and significant global rate of convergence $^{[1]}$.
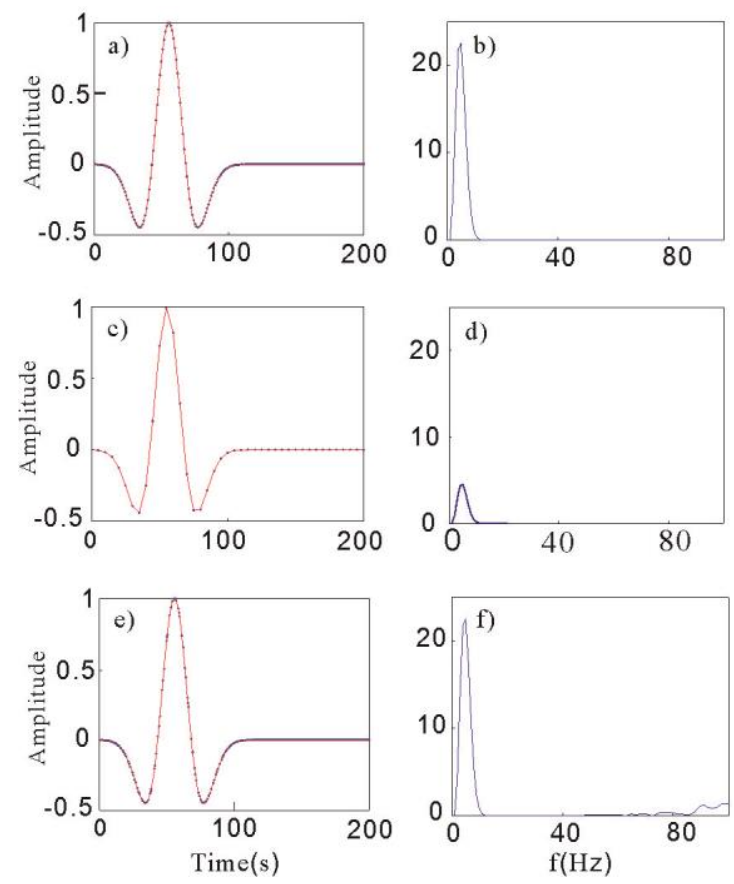

Fig. 1 The results of sampling and corresponding spectrum. a) Dense regular sampling; c) Sparse regular sampling; e) Dense irregular sampling; b), d) and f) are the frequency spectrum of a), b) and c), respectively 


\section{THEORY}

Actually, the seismic data reconstruction equation can be expressed as follows

$$
\mathbf{d}=\mathbf{A} \mathbf{u}+\mathbf{w},
$$

where $\mathbf{d} \in \square^{m}$ represents the observed data which is sparse sampled, $\mathbf{A} \in \square^{m \times n} \quad$ represents sampling matrix, $\mathbf{u} \in \square^{n}$ represents the "true" seismic data to be estimated, $\mathbf{W}$ represents an unknown noise vector. Equation (1) is an illposed equation due to $n>m$, that is to say, its solution is not unique.

A fundamental requirement is assuming that seismic data has a sparse representation in some known transform domain, such as Fourier domain and Curvelet domain. In Fourier domain, seismic data can be transformed to sparsity by:

$$
\mathbf{r}=\mathbf{F u},
$$

The operator $\mathbf{F}$ is Fourier transformation basis or dictionary which make $\mathbf{r}=\mathbf{F u}$ sparse. And $\mathbf{F}^{H} \mathbf{F}=I, I$ represents the unit matrix. As a result, equation (1) now can be reformulated as follows

$$
\mathbf{d}=\mathbf{M r}+\mathbf{w}, \mathbf{M}=\mathbf{A} \mathbf{F}^{H},
$$

Then, we need the coherence between sampling matrix $\mathbf{A}$ and transformation dictionary $\mathbf{F}$ is little. Therefore, the reconstructed seismic data $\mathbf{u}$ can be obtained by follow equation (Candes et al., 2006; Donoho, 2006; Hennenfent and Herrmann, 2008; Liu, 2015):

$$
\tilde{\mathbf{r}}=\arg \min _{r}\|\mathbf{r}\|_{1}, \text { s.t. }\|\mathbf{M r}-\mathbf{d}\|_{2} \leq \varepsilon,
$$

Then, to simplify the problem, we introduce the Lagrangian multiplier $\lambda$, equation (4) is equivalent to the following equation (Yuan and Wang, 2013)

$$
\tilde{\mathbf{r}}=\arg \min _{r} \frac{1}{2}\|\mathbf{M r}-\mathbf{d}\|_{2}^{2}+\lambda\|\mathbf{r}\|_{1},
$$

where $\lambda$ determines the weight of two items. Actually, $\lambda$ is a changing variable which influences the final optimal solution.

The fast iterative threshold shrinkage algorithm (FISTA) is used to solve equation (5).The constant algorithm flow is presented, as follows:

Step 0. Take $y_{1}=r_{0} t_{1}=1$;

Step k. $k \geq 1$ Compute (6) and (7)

$$
t_{k+1}=\frac{1+\sqrt{1+4 t_{k}^{2}}}{2},
$$

$$
y_{k+1}=r_{k}+\frac{t_{k-1}}{t_{k+1}}\left(r_{k}-r_{k-1}\right) \text {, }
$$

\section{EXAMPLES}

In this section, firstly, we use numerical model to test seismic data reconstruction method. Then we apply this method to reconstruction the field data which has no any processing.

First, synthetic of single layer subsurface model is generated to test the reconstruction method. The short gather is shown in Fig 2, which is with 200 traces, 1000 sampling points, $1 \mathrm{~ms}$ sampling interval and $8 \mathrm{~m}$ spatial sampling interval.

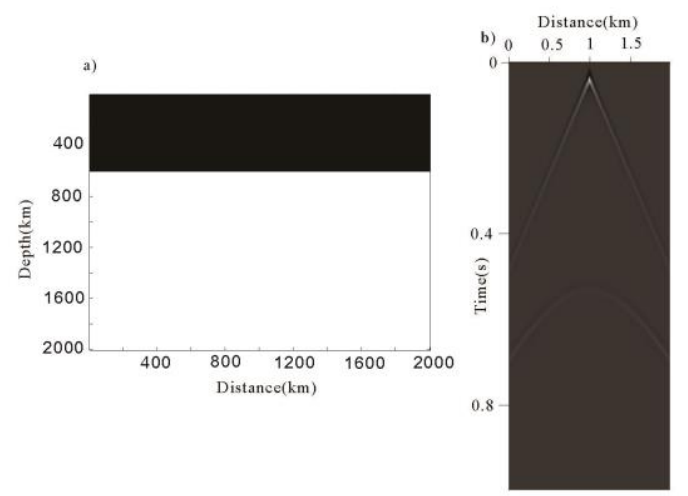

Fig. 2 Velocity model and original complete data. a) Single layer velocity model; b) Original seismic record

Fig 3a) and Fig 3c) show the incomplete with $19 \%$ and $65 \%$ traces randomly removed, respectively. And Fig $3 \mathrm{~b}$ ) and Fig 3d) show the reconstruction result corresponding to Fig 3a) and Fig 3c), respectively. It can be see that the missing seismic data is recovered successfully when random $19 \%$ traces are removed. Even to the random sampling $65 \%$ missing traces, the reconstruction result is acceptable.

Then, synthetic of Marmousi model is generated to test the reconstruction method. The short gather is shown in Fig 4, which is with 383 traces, 3001 sampling points, $1 \mathrm{~ms}$ sampling interval and $10 \mathrm{~m}$ spatial sampling interval. Fig 5a) and Fig 5c) show the incomplete with $19 \%$ and $65 \%$ traces randomly removed. And Fig 5b) and Fig 5d) show the reconstruction result corresponding to Fig 5a) and Fig 5c), respectively. It can be see that the missing seismic data is recovered successfully even to the complex model. 

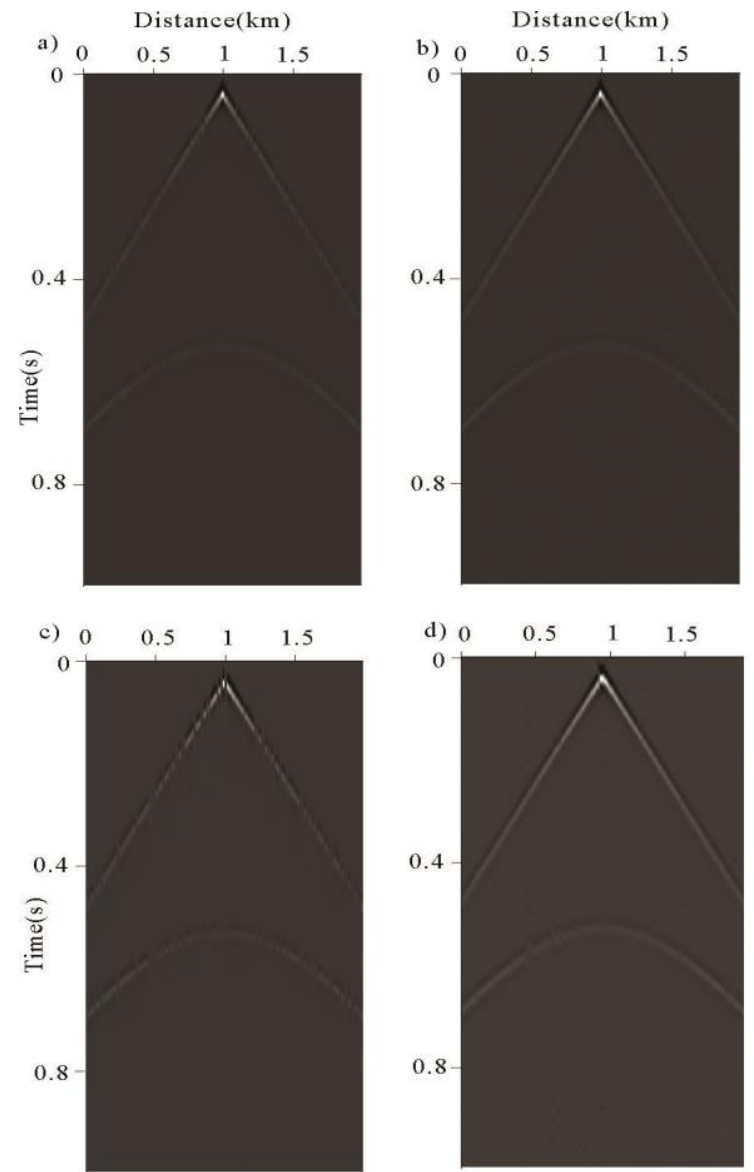

Fig. 3 Results of different sampling to single layer model. a) Random sampling 19\% missing traces; b) Reconstruction results corresponding to a); c) Random sampling $65 \%$ missing traces; b) Reconstruction results corresponding to d)
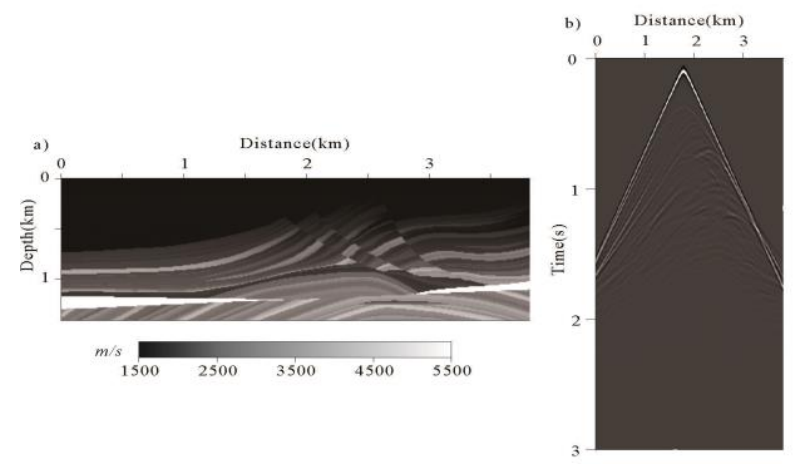

Fig. 4 Velocity model and original complete data. a) Marmousi velocity model; b) Original seismic record

For our second example, we apply real field data to test this reconstruction method. The observed data are with 180 traces, 2000 sampling points, $1 \mathrm{~ms}$ sampling interval and $5 \mathrm{~m}$ spatial sampling interval. Fig 6a) shows the seismic record of any shot, we randomly removed $15 \%$ traces. Fig 6b) shows the reconstruction result, it can be see that, though the result is acceptable, there exist some artifacts.

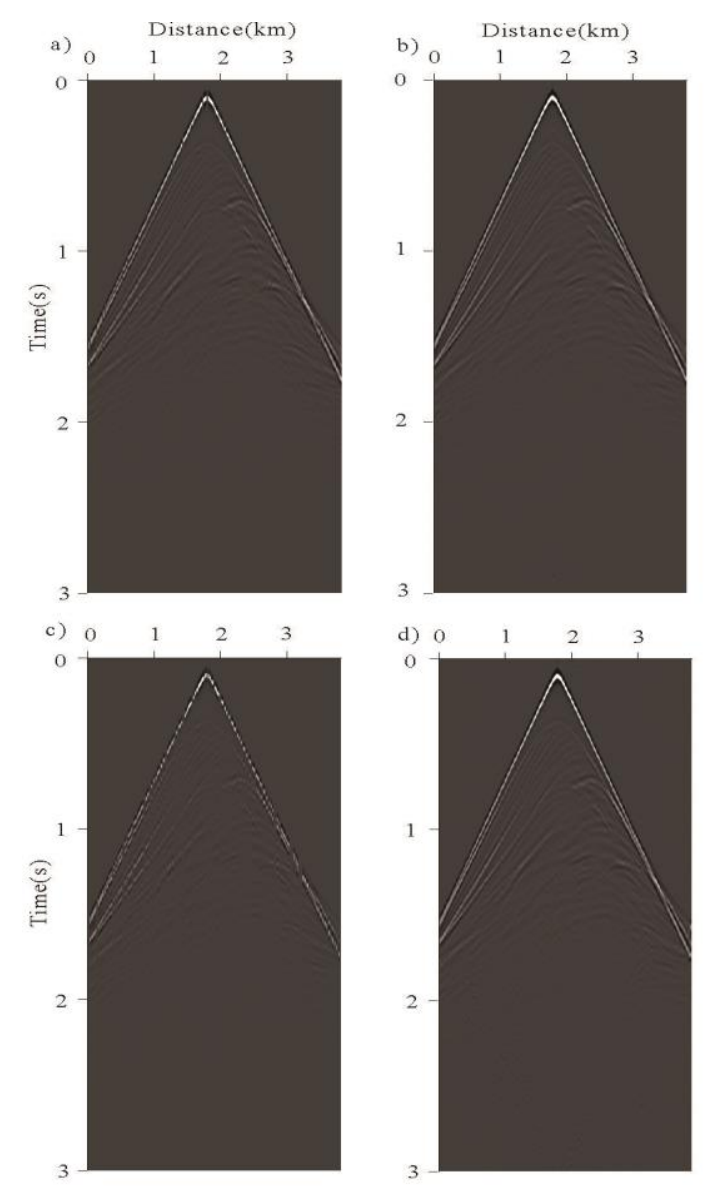

Fig. 5 Results of different sampling to Marmousi model. a) Random sampling $19 \%$ missing traces; b) Reconstruction results corresponding to a); c) Random sampling $65 \%$ missing traces; b) Reconstruction results corresponding to d)
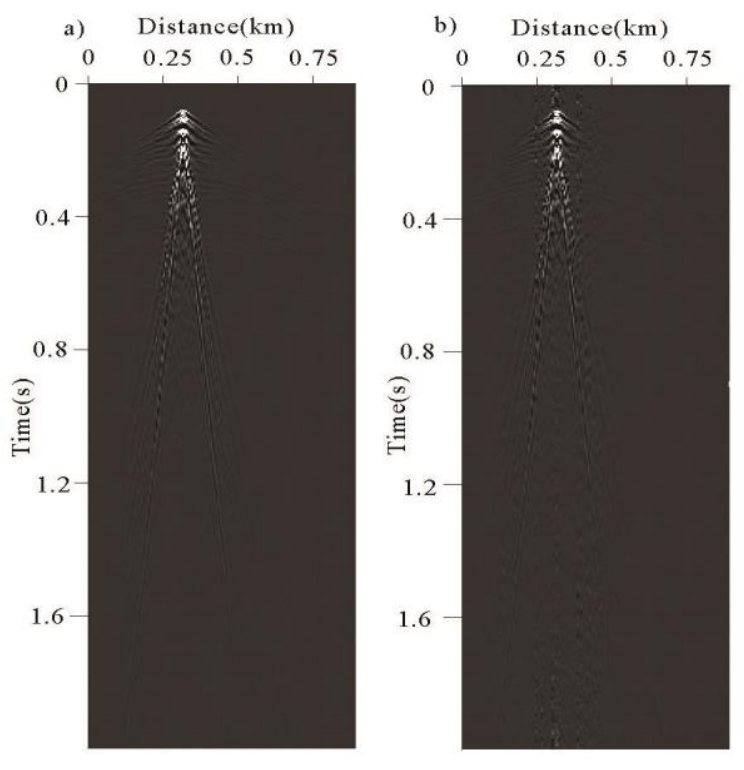

Fig. 6. Field data reconstruction result. a) Original field data; b) Reconstructed data form data with $15 \%$ randomly missing data 


\section{CONCLUSIONS}

In this paper, we have introduced a general seismic data reconstruction framework based on Compressed Sensing (CS) theory and Fourier transform, which is fast and efficient. We also find that sparsity assumption is necessary to make use of the theory of compressive sensing.

Through numerical modeling test and field test, we can find that the reconstruction method is significant. The seismic data is complete and continuous, which will benefit the following processing. Next, we need further study the seismic data reconstruction for field data which is more complex than modeling data.

\section{ACKNOWLEDGMENT}

This work is supported by the R\&D of Key Instruments and Technologies for Deep Resources Prospecting (the National R\&D Projects for Key Scientific Instruments), Grant NO. ZDYZ2012-1-06, and Open Issue of Key Laboratory of Mineral Resources, Chinese Academy of Sciences, Grant NO.KLMR2015-14.

\section{REFERENCES}

[1] Baraniuk, R.G.,2007, Compressive sensing :IEEE Signal Processing Magazine, 24, July,118-124.

[2] Candès, E. J., and M. B. Wakin, 2008, An introduction to compressive sampling: IEEE Signal Processing Magazine, 25, no. 2, 21-30.

[3] Candès, E. J., Demannet, S.L., Donoho, D.L., et al., 2006, Fast discrete curvelet transform. Multiscale Model ,No.5, 861-899.

[4] Candès, E. J., Donoho, D.L., 2004, New tight frames of curvelets and optimal representations of objects with piecewise- $C^{2}$ singularities: Common. Pure Appl. Math.57(2), 219-266.

[5] Chengbo Li, Charles C. Mosher and Sam T. Kaplan, ConocoPhillips, 2012, Interpolated compressive sensing for seismic data reconstruction: SEG Technical Program Expanded Abstracts 2012: pp. 1-6.

[6] Donoho, D., 2006, Compressed sensing: IEEE Transactions on Information Theory, 52, no. 4, 1289-1306.

[7] Ronen, J., 1987, Wave equation trace interpolation: Geophysics 52(7), 973-984.

[8] Sacchi M D, Ulrych T J, Walker C. Interpolation and exploration using a high-resolution discrete Fourier transform: IEEE Trans on Signal Process. 46 (1), 31-38.

[9] Spitz, S., 1991, Seismic trace interpolation in the F-X domain: Geophysics 56(6), 785-794.

[10] Wei Liu, Siyuan Gao, Guofa Li, and Yuan he, 2015, Reconstruction of seismic data with missing traces based on local random sampling and curvelet transform. Journal of Applied Geophysics: No.115, 129-139. 